ARTICLE

https://doi.org/10.1038/s41467-019-10484-7

\title{
Natural hypothalamic circuit dynamics underlying object memorization
}

\author{
Christin Kosse $^{1,2}$ \& Denis Burdakov (1) ${ }^{1,3}$
}

Brain signals that govern memory formation remain incompletely identified. The hypothalamus is implicated in memory disorders, but how its rapidly changing activity shapes memorization is unknown. During encounters with objects, hypothalamic melanin-concentrating hormone $(\mathrm{MCH})$ neurons emit brief signals that reflect object novelty. Here we show that targeted optogenetic silencing of these signals, performed selectively during the initial object encounters (i.e. memory acquisition), prevents future recognition of the objects. We identify an upstream inhibitory microcircuit from hypothalamic GAD65 neurons to MCH neurons, which constrains the memory-promoting $\mathrm{MCH}$ cell bursts. Finally, we demonstrate that silencing the GAD65 cells during object memory acquisition improves future object recognition through $\mathrm{MCH}$-receptor-dependent pathways. These results provide causal evidence that object-associated signals in genetically distinct but interconnected hypothalamic neurons differentially control whether the brain forms object memories. This gating of memory formation by hypothalamic activity establishes appropriate behavioral responses to novel and familiar objects.

\footnotetext{
${ }^{1}$ The Francis Crick Institute, London NW1 1AT, UK. ${ }^{2}$ Laboratory of Molecular Genetics, The Rockefeller University, New York, NY 10065, USA.

${ }^{3}$ Neurobehavioural Dynamics Lab, Institute for Neuroscience, D-HEST, Swiss Federal Institute of Technology / ETH Zürich, Zürich 8603, Switzerland.

Correspondence and requests for materials should be addressed to D.B. (email: denis.burdakov@hest.ethz.ch)
} 
T he ability to memorize objects enables one to react differently to novel and familiar objects. This ability is fundamental for normal life and impaired in many common and devastating brain pathologies ${ }^{1,2}$. It is still debated which brain structures and signals are critical for the formation of object memory ${ }^{3,4}$. Intense research into this topic traditionally focused on brain areas such as the perirhinal cortex and hippocampus ${ }^{2,4}$. In contrast, causal roles of neural dynamics of the hypothalamus have been underexplored, despite over half a century of evidence implicating this region in memory disorders ${ }^{5-9}$. The hypothalamus contains multiple neuronal types interconnected in complex and poorly-understood ways, including neurons expressing the peptide neurotransmitter melanin-concentrating hormone $(\mathrm{MCH})^{10}$ that innervate many brain areas thought to be important for memory control ${ }^{8,11}$. While originally $\mathrm{MCH}_{\mathrm{LH}}$ neurons were only thought to be active during sleep $^{12}$, it was found recently that they are also active during awake spatial exploration ${ }^{13}$. However, it remains unknown whether this natural $\mathrm{MCH}_{\mathrm{LH}}$ cell activity during wakefulness influences object memory formation, because wakefulness-specific silencing of $\mathrm{MCH}_{\mathrm{LH}}$ neurons in the context of object memorization has not been performed. The neural circuits shaping $\mathrm{MCH}_{\mathrm{LH}}$ cell activity during wakefulness and roles of such circuits in memory formation also remain undefined. Here we explored these unknowns by reversible silencing of $\mathrm{MCH}_{\mathrm{LH}}$ cells and their upstream neurons (newly identified here) during encounters with objects, combined with examining the subsequent behavioral reactions to novel and previously encountered objects.

\section{Results}

Natural MCH cell activity represents object novelty. Recordings of natural $\mathrm{MCH}_{\mathrm{LH}}$ cell activity during self-paced navigation in object-containing arenas revealed that $\mathrm{MCH}_{\mathrm{LH}}$ cells emitted activity bursts when mice encountered objects (Fig. 1a-f, Supplementary Fig. 1A-E; encounter was defined by real-time videotracking as an entry of mouse nose into the object area, see "Methods"). Such object-related activity was not observed in LH hypocretin/orexin neurons (Supplementary Fig. 1F), indicating a cell-type-specificity of LH responses to object encounters. When recorded continuously during sequential presentation of novel and familiar objects to the same mice, the novel-objectencounter-associated $\mathrm{MCH}_{\mathrm{LH}}$ signals decreased as mice spent more time with the object (Fig. 1d-h; Supplementary Fig. 1E), but increased again when they were presented with a new novel object (Fig. 1d, e; Supplementary Fig. 1C). When mice were presented with familiar objects, the object-encounter-associated $\mathrm{MCH}_{\mathrm{LH}}$ signals during consecutive object encounters tended to remain small in amplitude, in contrast to novel-object-associated signals that were initially large and decayed during consecutive object encounters (Fig. 1f-h). This object familiarization-evoked reduction in $\mathrm{MCH}_{\mathrm{LH}}$ signals persisted when the familiar object was moved to a new location (Supplementary Fig. 1B, D), and was maintained for up to $20 \mathrm{~h}$ (Supplementary Fig. 1C, D). Together, these properties of object-encounter-associated $\mathrm{MCH}_{\mathrm{LH}}$ signals are consistent with signals associated with object memorization.

Causal link of natural MCH cell dynamics and object memory. To probe whether the object encounter-associated signals of $\mathrm{MCH}_{\mathrm{LH}}$ cells play a causal role in object memory formation, we close-looped real-time video-tracking of object encounters to $\mathrm{MCH}_{\mathrm{LH}}$ cell optosilencing in $\mathrm{MCH}_{\mathrm{LH}}:$ :ArchT mice (Fig. $2 \mathrm{a}-\mathrm{c}$, see "Methods"). We did this in the context of a classic object memory tes $^{14,15}$, where mice are exposed to pairs of objects in 2 temporally separated trials (Fig. 2c). In trial 1 (memory acquisition phase) they encountered two identical novel objects, with or without the object-associated $\mathrm{MCH}_{\mathrm{LH}}$ cell optosilencing (Fig. 2c). In trial 2 (object recognition test), which involved no optosilencing, object memory was quantified as time spent with a novel vs. the previously encountered object (Fig. 2c, this quantifies object memory since in this test mice are normally less drawn to previously encountered objects ${ }^{14,15}$ ). To prevent variations in sensory or laser exposure from affecting memorization, we tracked the total object encounter time in trial 1 , and matched its value across compared conditions (see "Methods" section "Object recognition tests with controlled familiarization time"). $\mathrm{MCH}_{\mathrm{LH}}$ cell optosilencing selectively during object encounters in trial 1 prevented $\mathrm{MCH}::$ ArchT mice from recognizing the previously encountered objects in trial 2 (Fig. 2d). In contrast, trial 2 object recognition was normal when the same closed-loop LH laser illumination experiment was performed in control mice lacking the optoinhibitory opsin $\left(\mathrm{MCH}_{\mathrm{LH}}: \mathrm{GCaMP}\right.$ mice) (Fig. 2d). This shows that the natural $\mathrm{MCH}_{\mathrm{LH}}$ cell activity during initial object encounters is necessary for the object to be treated as familiar in the future, i.e., for object recognition memory formation. The controlled design of these experiments (exposure time matching, mixed order within-mouse repeats, control mice, see "Methods") indicates that the disruption of object memory formation by the temporally-targeted optosilencing of $\mathrm{MCH}_{\mathrm{LH}}$ cells was not due to differences in sensory exposure, or order or laser-related effects.

Identification of inhibitory GAD65 $\rightarrow \mathrm{MCH}$ neural microcircuit. The above findings show that inhibition of the object-associated $\mathrm{MCH}_{\mathrm{LH}}$ activity selectively during memory acquisition is a powerful way to control object memory formation. In search for neural origins of this inhibition, we used channelrhodopsin (ChR2)assisted circuit mapping to probe functional interactions of $\mathrm{MCH}_{\mathrm{LH}}$ and neighboring non-MCH cells with local $\mathrm{GAD} 65_{\mathrm{LH}}$ neurons, a recently-characterized $\mathrm{LH}$ neural type whose downstream cell targets are yet unknown ${ }^{16}$. In mouse brain slices, optostimulation of GAD65 ${ }_{\mathrm{LH}}:$ :ChR2 cells evoked rapid GABAergic inhibitory input in $\mathrm{MCH}_{\mathrm{LH}}$ cells (Fig. 3a) but not in the neighboring LH orexin/ hypocretin cells (Supplementary Fig. 3B). Optostimulation of $\mathrm{MCH}_{\mathrm{LH}}::$ ChR2 cells did not evoke detectable input in GAD65 $5_{\mathrm{LH}}$ cells (Supplementary Fig. 3A), suggesting a unidirectional GAD65 $\rightarrow \mathrm{MCH} \mathrm{LH} \mathrm{microcircuit.} \mathrm{In} \mathrm{complementary} \mathrm{in} \mathrm{vivo}$ circuit-connectivity screens in object-exploring mice, chemogenetic activation of GAD65 $5_{\mathrm{LH}}: \mathrm{hM} 3 \mathrm{Dq}$ cells (Fig. 3b, see "Methods") was able to suppress the novel object encounter-associated activity $\mathrm{MCH}_{\mathrm{LH}}$ cell bursts (Fig. 3c, d). Thus, a functional inhibitory GAD65 $\rightarrow \mathrm{MCH} \mathrm{LH}$ circuit exists that is sufficiently powerful to suppress object-encounter-associated $\mathrm{MCH}_{\mathrm{LH}}$ cell activity.

Natural GAD65 cell activity controls memory via $\mathrm{MCH}$ pathways. To investigate whether the natural $\mathrm{GAD} 65_{\mathrm{LH}}$ cell activity influences object memory acquisition via the $\mathrm{MCH}$ system, we repeated the memory acquisition-coupled optogenetic interference (Fig. 2) with $\mathrm{GAD} 65_{\mathrm{LH}}$ optosilencing. The GAD65 $_{\mathrm{LH}}$ cell optosilencing targeted to object encounters during object memory acquisition significantly increased subsequent novel object preference during object recognition (Fig. 4c, d). This indicates that the natural activity of GAD65 $5_{\mathrm{LH}}$ cells opposes object memory formation, as expected from the inhibitory GAD65 $\rightarrow \mathrm{MCH}$ LH circuit. If this effect of GAD65 ${ }_{\mathrm{LH}}$ cells on object memory formation is mediated by the inhibitory GAD65 $\rightarrow \mathrm{MCH} \mathrm{LH}$ circuit, then it should be diminished by blocking MCH cell outputs. Consistent with this prediction, when the GAD65 $5_{\mathrm{LH}}$ cell optosilencing was performed concurrently with $\mathrm{MCH}$ receptor blockade using the $\mathrm{MCH}$ receptor antagonist SNAP94847 $(20 \mathrm{mg} / \mathrm{kg}$ i.p., see "Methods"), the effect of the GAD65 ${ }_{\mathrm{LH}}$ cell optosilencing was 
a

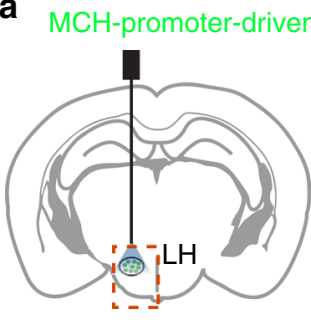

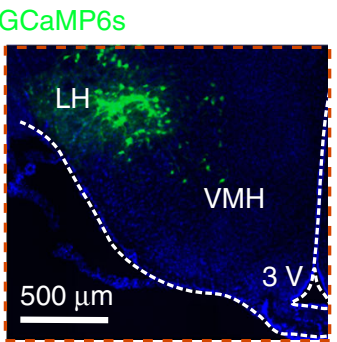

b

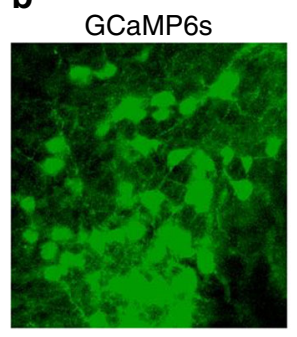

$\mathrm{MCH}$ antibody

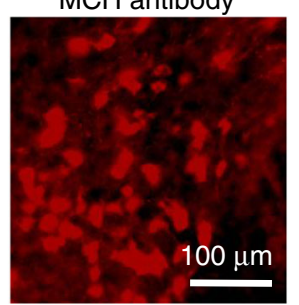

Overlay

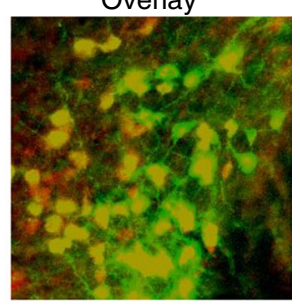

C

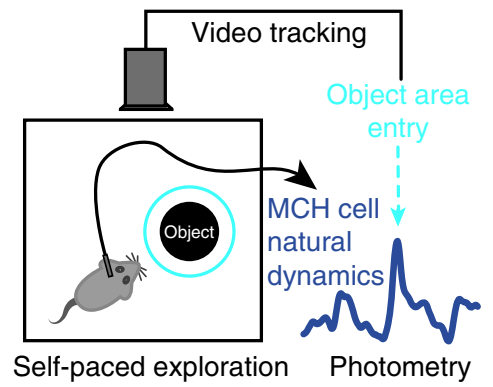

f

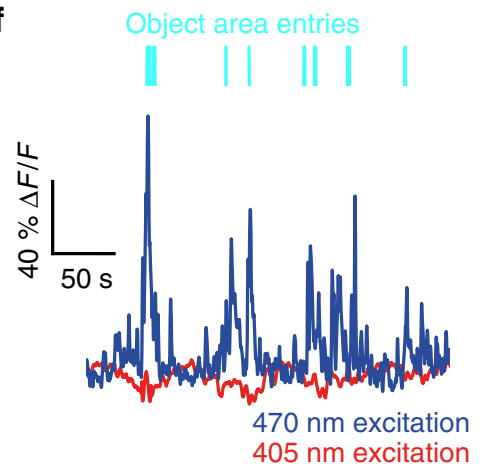

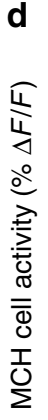

g

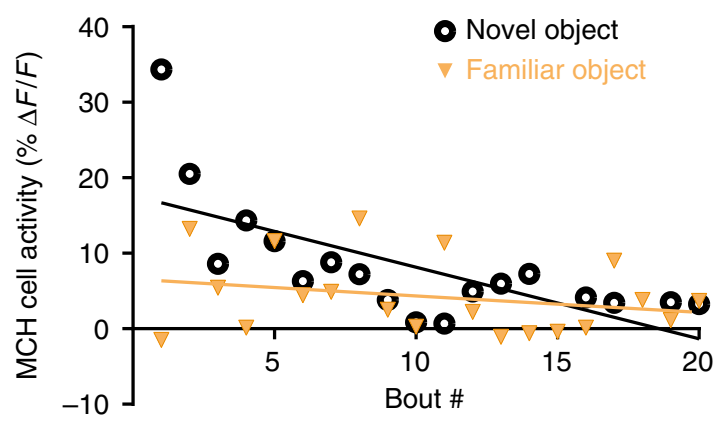

Familiar object

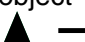

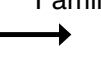
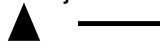

Novel object 2
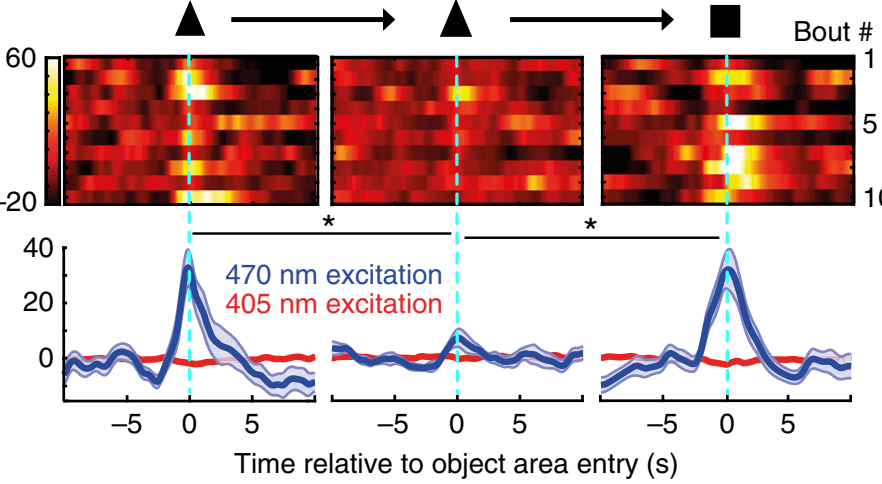

e

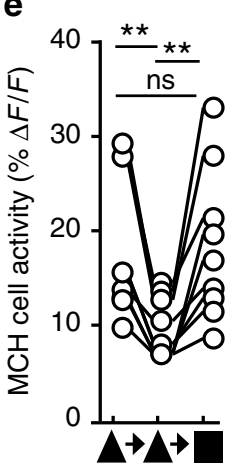

Fig. 1 Representations of novel objects in $\mathrm{MCH}$ cell dynamics. a Targeting scheme (left) and expression (right) of $\mathrm{GCaMP}_{\mathrm{S}}$ in $M C \mathrm{H}_{\mathrm{LH}}$ cells. b Confirmation of GCaMP6s expression in $\mathrm{MCH}_{\mathrm{LH}}$ cells (see "Methods"). c Schematic (left) of $\mathrm{MCH}_{\mathrm{LH}}$ cell recording concurrent with behavioral tracking. d Top, representative heatmaps of $\mathrm{MCH}_{\mathrm{LH}}:$ GCaMP6s fluorescence (at $470 \mathrm{~nm}$ excitation) aligned to object-area entry. First 10 object area entries from one mouse (representative data of $n=9$ mice). Bottom, group data (means \pm s.e.m of $n=10$ objects visits for one mouse), also showing negative control from $405 \mathrm{~nm}$ excitation. One-way RM ANOVA $\mathrm{F}(2,27)=5.505, p=0.0099$, Tukey's multiple comparisons: Novel object 1 (triangle) vs novel object: ${ }^{*} p=$ 0.0227 , Novel object vs. novel object 2 (rectangle): ${ }^{*} p=0.0189$. e Quantification of peak $M_{C H} H_{L H}$ cell activity from $D, n=9$ mice. One-way RM ANOVA $F$ $(1.694,13.55)=16.52, p=0.0004$, Tukey's multiple comparisons post-tests: novel object 1 (triangle symbol) vs novel object 2 (square symbol) $p=0.9991$ (ns), novel object 1 vs familiar object (same object presented 30 min later) ${ }^{\star \star} p=0.0021$, familiar object (middle triangle symbol) vs novel object 2 (square symbol): ${ }^{\star \star} p=0.0073$. f Representative $\mathrm{MCH}_{\mathrm{LH}}$ cell responses from one mouse to a sequence of self-paced novel object area entries. $\mathbf{g}$ Representative peak $\mathrm{MCH}_{\mathrm{LH}}$ cell responses from one mouse to a self-paced sequence of novel (NO) and familiar (FO) object area entries; straight lines are linear fits to the data. $\mathbf{h}$ Quantification of data in $\mathrm{G}$ for $n=9$ mice, paired t-test: ${ }^{\star \star \star} p=0.0074, t(8)=3.556$

abolished (Fig. 4c, d). Conversely, object memory formation driven by natural $\mathrm{MCH}$ signaling (isolated by quantifying behavior with and without SNAP94847 in individual mice) was significantly increased by GAD65 ${ }_{\mathrm{LH}}$ cell inhibition (Fig. 4e), confirming that $\mathrm{MCH}_{\mathrm{LH}}$ cells regulate behavior according to GAD65 $5_{\mathrm{LH}}$ cell tone. This shows that the object-encounterassociated, natural GAD65 $5_{\mathrm{LH}}$ cell activity governs object memory formation via MCH-receptor-dependent pathways.

\section{Discussion}

Our findings indicate that mice do not recognize objects unless their $\mathrm{MCH}$ cells are active during the prior object encounters, and that a novel GAD65 $\rightarrow \mathrm{MCH}$ microcircuit governs the size of the memory-controlling $\mathrm{MCH}$ cell bursts. The type of object recognition memory that we studied is fundamentally important for normal behavior in both humans and animals ${ }^{2,17}$. To the best of our knowledge, our study is the first to make causal links between the transient, object-associated dynamics of hypothalamic neurons and memory formation. Previous molecular and pharmacological studies linked $\mathrm{MCH}$ peptide signaling to avoidance memory $8,9,18,19$, but did not involve temporally precise manipulation of ongoing $\mathrm{MCH}$ cell activity at behaviorallyrelevant timescales, and thus contained no indication when the natural $\mathrm{MCH}$ cell activity influences memory, nor how upstream circuits shape these memory-gating $\mathrm{MCH}$ signals. Our data thus provide causal evidence for the role of object-associated signals in a hitherto unknown hypothalamic circuit in object recognition memory formation. 
a

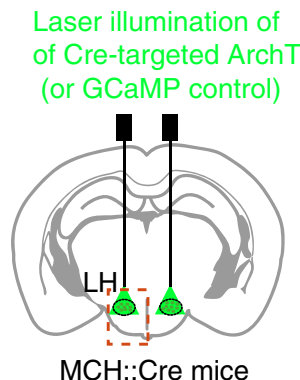

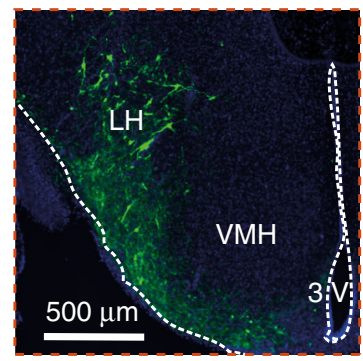
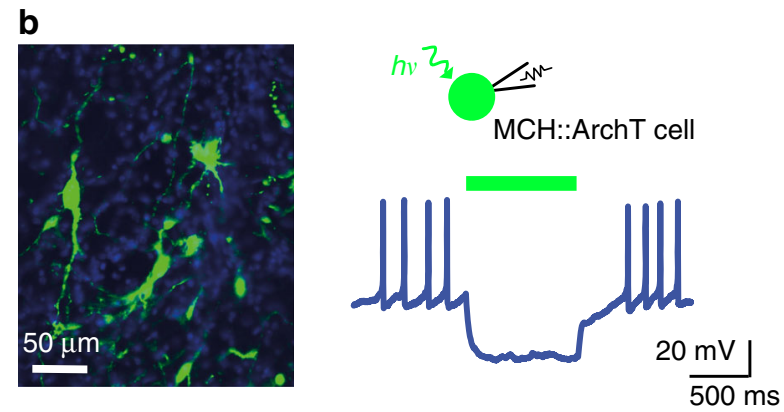

C

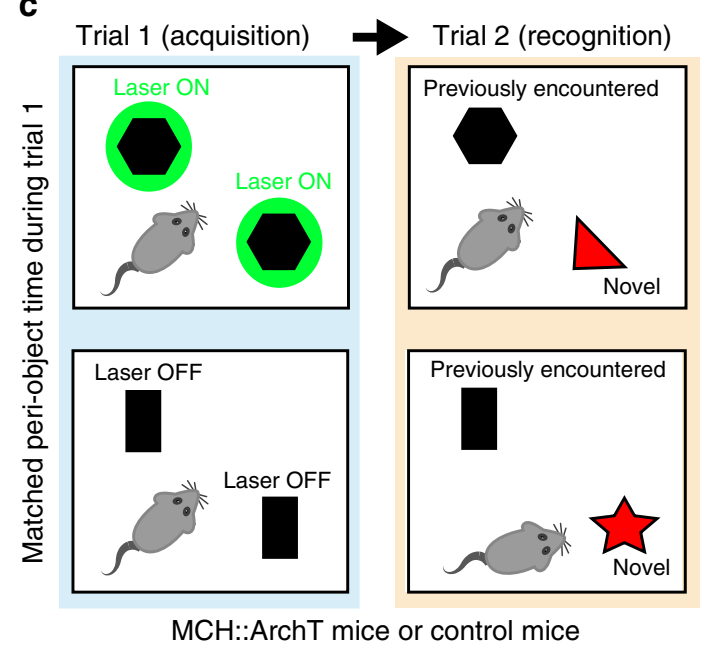

d Recognition behavior +/- acquisition-restricted optosilencing After Trial 1 laser ON

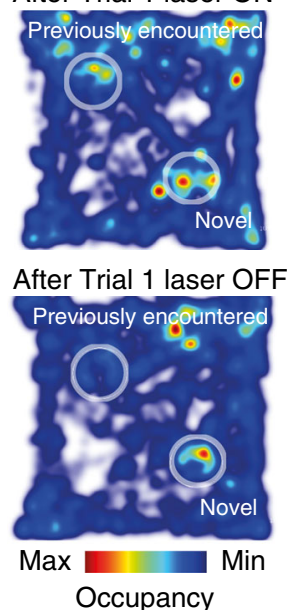

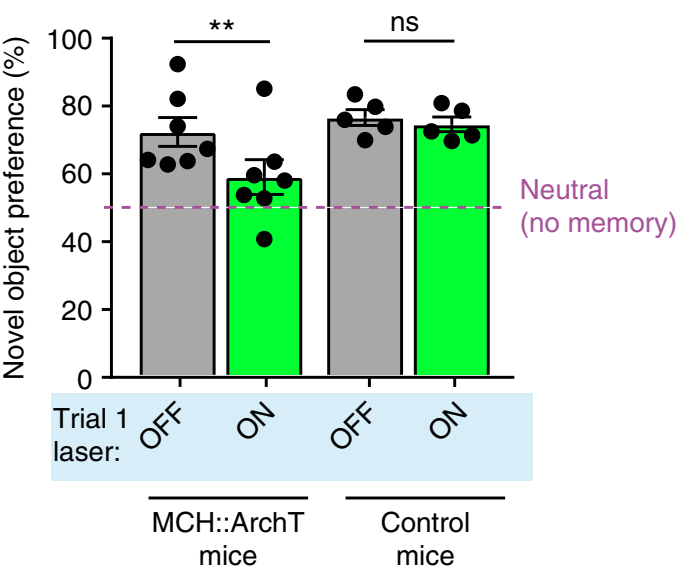

Fig. 2 Natural MCH cell bursts gate object memory formation. a Targeting scheme (left) and expression (right) of ArchT in $M C H_{L H}$ cells. b Patch-clamp recording (right) confirming silencing of $\mathrm{MCH}_{\mathrm{LH}}:$ :ArchT-YFP cells (left) by green light ( $n=5$ cells). c Experimental scheme: self-paced exploration of two identical novel objects for the same cumulative peri-object time (trial 1) followed by quantifying exploration of the same arena with one of the previously encountered objects replaced by a novel object (trial 2). $\mathbf{d}$ Left: sample heatmaps showing relative time spent with objects on trial 2. Right: group data (individual points and their means \pm s.e.m). Object-area-entry-coupled bilateral LH laser illumination during trial 1 reduced object recognition in trial 2 in $\mathrm{MCH}_{\mathrm{LH}}:$ ArchT mice $(n=7)$ but not in control $\left(\mathrm{MCH}_{\mathrm{LH}}:: \mathrm{GCaMP}\right)$ mice $(n=5)$ (2-way ANOVA: $F(1,8)=7.43, p=0.0260$, Sidak's multiple comparisons

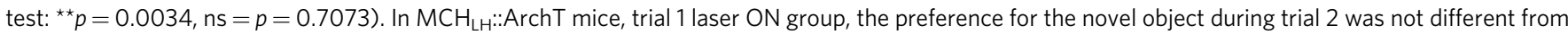
"no memory" (neutral, $50 \%$ ) criterion (one sample t-test against $50 \%$ preference: $t(6)=1.775, p=0.1262$ ), whereas in all other groups significant preference was seen (one sample t-tests against 50\% preference: $\mathrm{MCH}_{\mathrm{LH}}:$ ArchT mice laser OFF, $t(6)=5.276, p=0.0019$; control mice laser OFF, $\mathrm{t}(4)=$ 11.37, $p=0.0003$; control mice laser $\mathrm{ON}, \mathrm{t}(4)=11.42, p=0.0003$ )

These results support broader roles of rapid hypothalamic signals in cognition than previously considered, as also supported by recent data on other hypothalamic neurons ${ }^{20-23}$. Furthermore, our data suggest that the hypothalamus not only relays a critical input for computation of appropriate behavior, but that local hypothalamic microcircuits also contribute to this computation. This contribution can be critical for fundamentally important behavior, since disrupting natural LH processing transiently and specifically during initial object encounters prevented mice from displaying normal behavioral responses (i.e., recognition) to novel and familiar objects in the future (Figs. 2 and 4). While our study focussed on inanimate objects in order to isolate object memory effects from reward or social motivators, in the future it will be interesting to investigate how local hypothalamic processing affects novelty and familiarity behavior towards more complex objects such as food and conspecifics.

Our finding that GAD65 $\rightarrow \mathrm{MCH} \mathrm{LH}$ circuit is important for object memory does not rule out that this circuit may also be involved in other functions. While so far we found no evidence that $\mathrm{MCH}_{\mathrm{LH}}$ or GAD65 $\mathrm{LH}$ cells are involved in spatial working memory (Supplementary Fig. 4), nor that $\mathrm{MCH}$ cells signal spatial locations (Supplementary Fig. 1B, D), we did observe that they may signal novel sensory qualities of food (Supplementary Fig. 5). The possibility that this circuit may signal multiple novel sensory experiences does not undermine the validity and importance of our findings relating to its involvement in object recognition memory. At the neuroanatomical level, $\mathrm{MCH}_{\mathrm{LH}}$ cell axons and $\mathrm{MCH}$ receptors are found brain-wide, including multiple regions speculated to be involved in object memory $3,4,9,11,24$, where $\mathrm{MCH}$ is proposed to alter synaptic plasticity thus making memories more likely to form ${ }^{8,9}$. Probing this broader downstream connectivity of the GAD65 $\rightarrow$ $\mathrm{MCH}$ LH circuit will improve our understanding of hypothalamic gating of cognition, though this is likely to be challenging given the breadth of $\mathrm{MCH}_{\mathrm{LH}}$ projections and the current lack of consensus about relative roles played by different brain regions in object recognition memory. Upstream, it would be interesting to probe whether known regulatory inputs to $\mathrm{MCH}_{\mathrm{LH}}$ and $\mathrm{GAD65} 5_{\mathrm{LH}}$ cellsfor example orexin, insulin, and glucose $\mathrm{e}^{13,16,25,26}$ - may act to match memory-related processes to stress and energy levels.

In summary, our study identifies a neural circuit that governs brain representations of object novelty, and links the natural object-related activity of this circuit to a vital cognitive function: object recognition memory formation. This circuit mechanism for the control of object memory formation offers new insights 
a

Cre-inducible ChR2-eYFP $\mathrm{MCH}$ promoter-driven mCherry

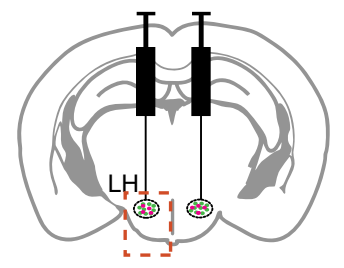

GAD65::Cre mice
Optogenetic identification of GAD65 $\rightarrow \mathrm{MCH}$ inhibitory circuit

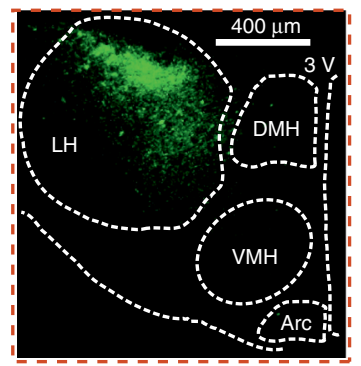

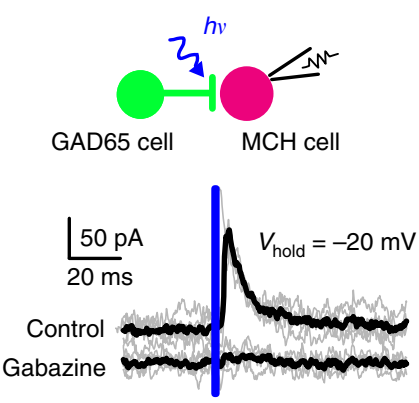

b Object-associated $\mathrm{MCH}$ cell dynamics during chemogenetic activation of GAD65 cells

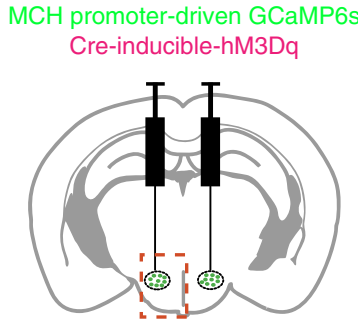

GAD65::Cre mice
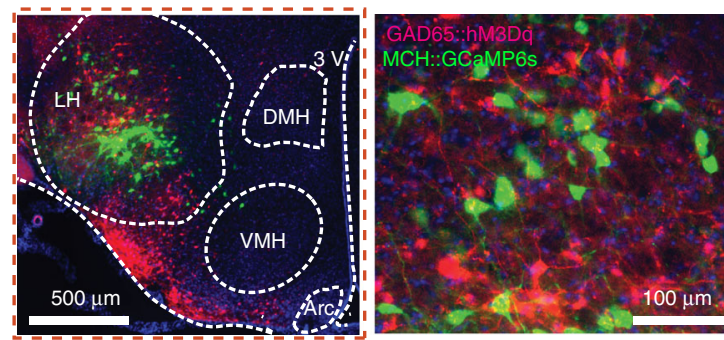

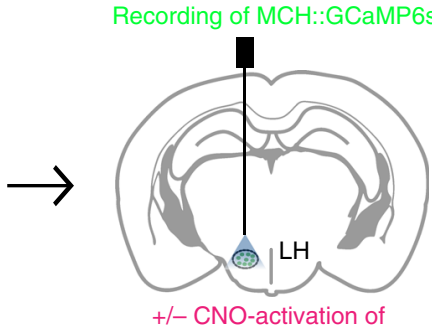

LH GAD65::hM3Dq cells
C

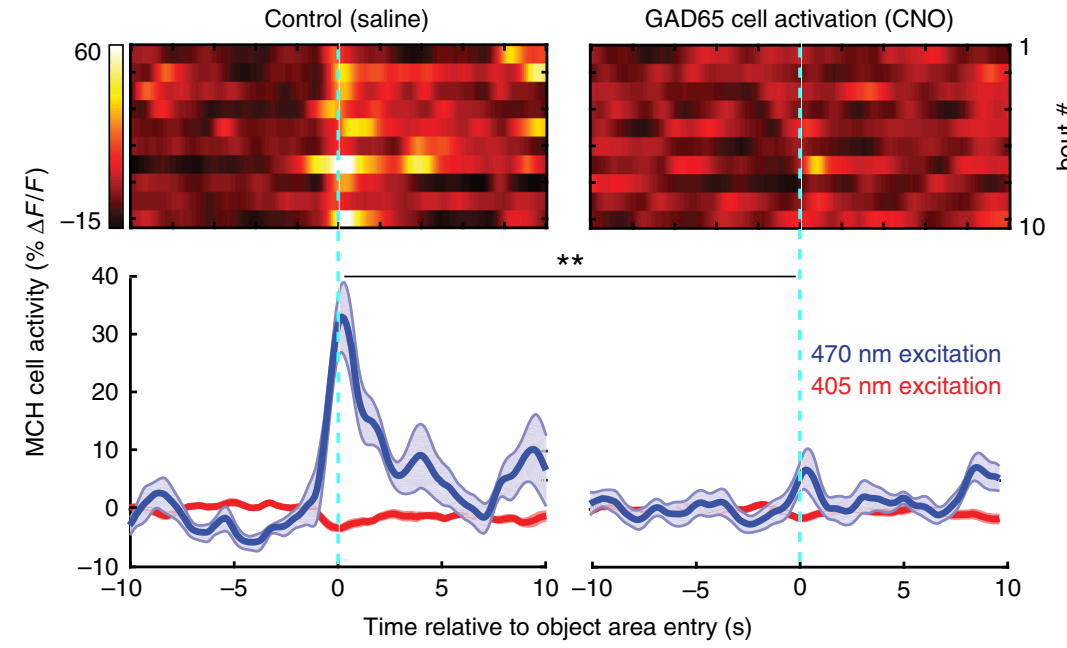

d

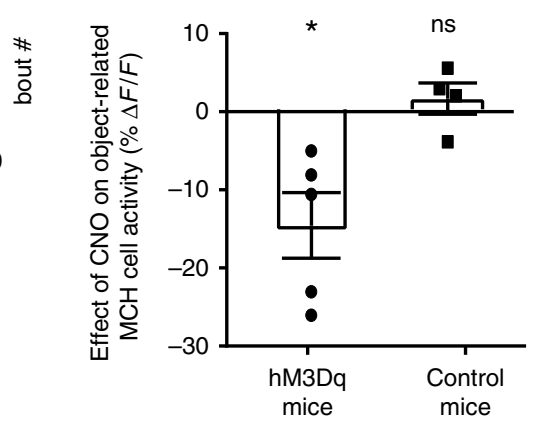

Fig. 3 Identification of GAD65 $\rightarrow$ MCH inhibitory microcircuit. a Targeting schematic (left) for expression of $\mathrm{ChR}_{2}$ in $\mathrm{GAD65} \mathrm{LH}_{\mathrm{L}}$ cells and mCherry in $\mathrm{MCH}_{\mathrm{LH}}$

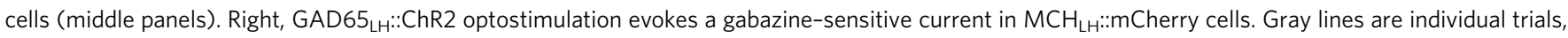
black lines are trial averages; $n=14 / 16$ cells were connected; latency between GAD65 LH cell optostimulation and the onset of postsynaptic current: $0.83 \pm$ $0.8 \mathrm{~ms}$ ( $n=14$ cells), synaptic current size is quantified in Supplementary Fig. 3C). b Targeting schematic (left) and expression (two middle panels) of GCaMP6s in $\mathrm{MCH}_{\mathrm{LH}}$ cells and hM3Dq in GAD65 ${ }_{\mathrm{LH}}$ cells, for recording of $\mathrm{MCH}_{\mathrm{LH}}:: G C a M P 6 s$ cell activity during GAD65 $\mathrm{LH}:$ :hM3Dq cell modulation (right). c Top, representative heatmaps of $\mathrm{MCH}_{\mathrm{LH}}:: \mathrm{GCaMP6s}$ fluorescence (at $470 \mathrm{~nm}$ excitation) aligned to object-area entry. First 10 object area entries from one mouse (representative data of $n=5$ mice). Bottom, corresponding group data (means \pm s.e.m of $n=10$ visits), also showing negative control from $405 \mathrm{~nm}$ excitation. Chemogenetic activation of GAD65 $\mathrm{LH}:$ hM3Dq cells decreased object-area-entry-associated $\mathrm{MCH} \mathrm{LH}_{\mathrm{H}}$ cell activity peaks ( $(18)=3.805$, ${ }^{\star \star} p=0.0013$, unpaired $t$-test, representative data comparing 10 object encounters before and after CNO from one mouse, group data are given in d). The late activity at around 5 and $10 \mathrm{~s}$ reflect activity outside the object area, which was not investigated further. $\mathbf{d}$ Group data (individual points and means $\pm \mathrm{s}$. e.m), showing effect of $\mathrm{CNO}$ (i.e., response in $\mathrm{CNO}$ minus response in saline) on peak peri-object $\mathrm{MCH}_{\mathrm{LH}}$ cell signals in negative control mice $\left(\mathrm{MCH}_{\mathrm{LH}}:\right.$. GCaMP6s, $n=4)$, and in hM3Dq mice $\left(\mathrm{MCH}_{\mathrm{LH}}:: \mathrm{GCaMP6s}\right.$ and GAD65 $\left.\mathrm{LH}:: \mathrm{hM} 3 \mathrm{Dq}, n=5\right) ;{ }^{\star} p=0.0257, t(4)=3.466$, one-sample $t$-test, $n=5$ mice; ns $=$ $p=0.4623, t(3)=0.8406$, one-sample $t$-test, $n=4$ mice

into neuromodulation of valued cognitive abilities that are key targets of rehabilitation in neuropsychiatric disease.

\section{Methods}

Genetic targeting. All procedures followed United Kingdom Home Office regulations and were approved by the Animal Welfare and Ethical Review Panel of the Francis Crick Institute. Mice were kept on a standard 12-h/12-h light/dark cycle and on standard mouse chow and water ad libitum. Adult male and female mice (at least 8-week old) were used for in vitro experiments. Adult male mice were used for behavioral experiments, which were performed during the dark phase. The following previously characterized and validated transgenic mouse lines (or their crosses) were used, where indicated: $\mathrm{MCH}:: \mathrm{Cre} \mathrm{mice}^{27}$, GAD65::Cre mice ${ }^{28}$, GAD65::GFP mice ${ }^{29}$, orexin::GFP mice ${ }^{30}$. The GAD65::Cre mice were bred in homozygous (hom)-WT pairs with C57BL/6 mice; all other transgenic mice were bred in het-WT pairs with C57BL/6 mice. For brain surgeries, mice were anesthetized with isoflurane and injected with meloxicam ( $2 \mathrm{mg} / \mathrm{kg}$ of body weight, s.c.) for analgesia. After placement into a stereotaxic frame (David Kopf Instruments), a craniotomy was performed and a borosilicate glass pipette was used to inject viral vectors bilaterally into the $\mathrm{LH}$. Two injections (each $75 \mathrm{~nL}$ ) were made into the $\mathrm{LH}$ in each hemisphere (bregma: $-1.30 \mathrm{~mm}$, midline: $\pm 1 \mathrm{~mm}$, from brain surface: $5.20 \mathrm{~mm}$ and $5.25 \mathrm{~mm}$ ). Before any manipulations, mice were allowed to recover 
a

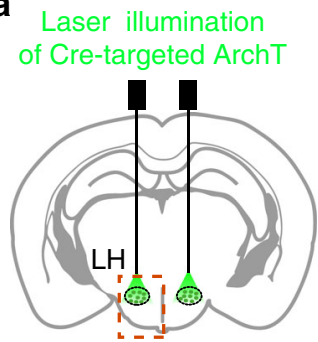

GAD65::Cre mice

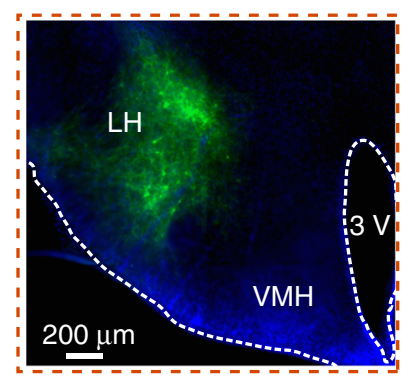

C

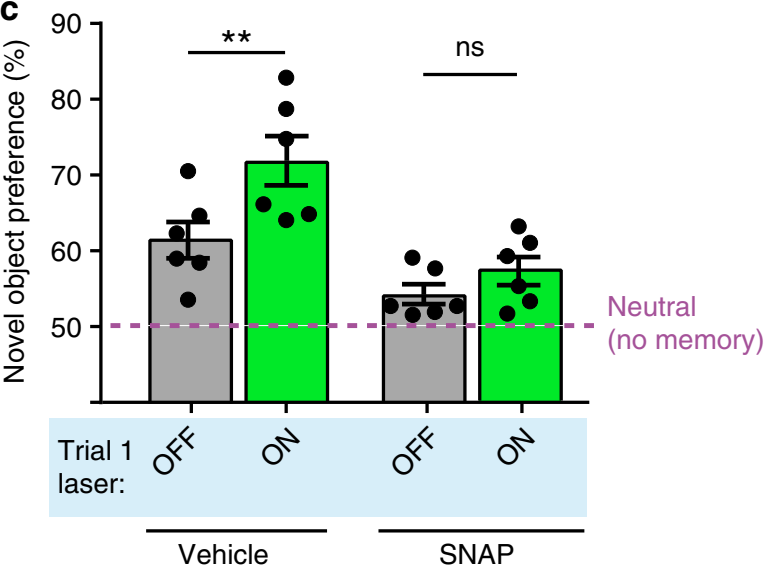

b
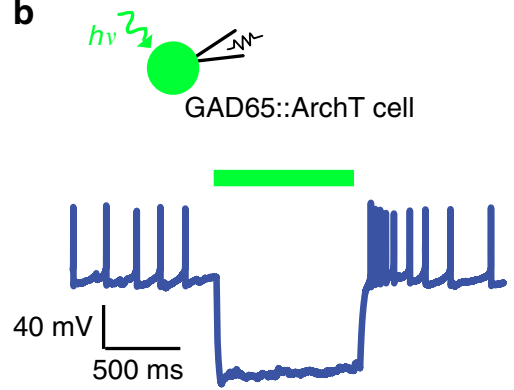

d

$\mathbf{e}$

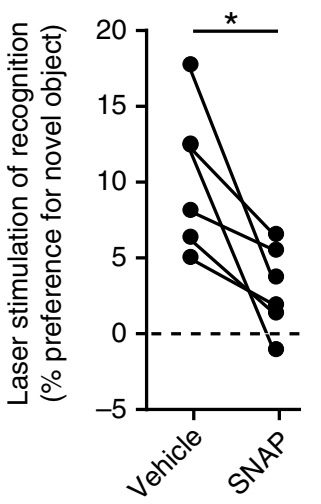

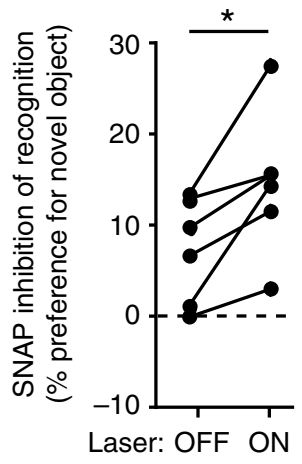

Fig. 4 GAD65 $5_{L H}$ cells control object memory via MCH signaling. a Targeting schematic (left) and expression (right) of ArchT in GAD65 $5_{L H}$ cells. b Patchclamp recording from GAD65 ${ }_{L H}:$ ArchT cells confirming cell inhibition by green light ( $n=5$ cells). c Role of GAD65 formation (experimental design as in Fig. 2C). Data are shown as individual points (mice) and their means \pm s.e.m. Object-area-entry-associated GAD65 ${ }_{\mathrm{LH}}$ cell optosilencing during trial 1 increased object recognition in trial 2 in the absence (2-way ANOVA, $F(1,5)=17.1, p=0.0090$, Tukey's multiple comparisons test: ${ }^{\star *} p=0.0036$ ) but not presence of MCHR blocker SNAP94847 (SNAP) (2-way ANOVA, $F(1,5)=17.1, p=0.0090$, Tukey's multiple comparisons test: $\mathrm{ns}=p=0.2931), n=6$ mice. $\mathbf{d}$ Quantification of trial 2 object recognition enhancement by the trial $1 \mathrm{GAD} 65_{\mathrm{LH}}$ cell optosilencing, in the presence and absence of SNAP ( $n=6$ mice, paired $t$-test: $\left.t(5)=3.488,{ }^{*} p=0.0175\right)$. e Quantification of trial 2 object recognition enhancement by the trial 1 SNAP, with and without concurrent GAD65 $\mathrm{LH}$ cell optosilencing, $n=6$ mice, paired $t$-test: $t(5)=3.488,{ }^{*} p=0.0175$

from surgery for at least 1 week while single-housed. To target expression of the activity indicator GCaMP6s to $\mathrm{MCH}_{\mathrm{LH}}$ neurons, we used an AAV vector carrying the $0.9 \mathrm{~kb}$ preproMCH gene promoter ${ }^{31}$, AAV9.pMCH.GCaMP6s.hGH $\left(1.78 \times 10^{14} \mathrm{gc} / \mathrm{mL}\right.$; Vigene Biosciences). The specificity of GCaMP6s expression was confirmed by staining with $\mathrm{MCH}$ antibody (Fig. 1b, $93 \%$ specificity was observed by analysis of MCH immunoreactivity colocalisation in 426 GCaMP6s neurons from three brains). For optogenetic silencing of $\mathrm{MCH}_{\mathrm{LH}}$ or $\mathrm{GAD} 65_{\mathrm{LH}}$ neurons, we injected Cre-dependent AAV8.Flex-ArchT-GFP $\left(4.6 \times 10^{12} \mathrm{gc} / \mathrm{ml}\right.$; UNC Vector Core) into LH of the MCH::Cre or GAD65::Cre mice, respectively. For ChR-assisted circuit mapping, "FLEX switch" ChR2 constructs were injected into LH of the MCH::Cre or GAD65::Cre mice, as indicated. These constructs were either AAV1.EF1.flox.hChR2(H134R)-mCherry.WPRE.hGH $\left(8.78 \times 10^{12} \mathrm{gc} / \mathrm{mL}\right.$; UPenn Vector Core) or AAV1.EF1.DIO.hChR2(H134R)-YFP.WPRE.hGH $\left(6.2 \times 10^{12} \mathrm{gc} / \mathrm{mL}\right.$; UPenn Vector Core). Cre-dependent "DREADD" chemogenetic actuator $\mathrm{hM} 3 \mathrm{Dq}$ was targeted to GAD65 $5_{\mathrm{LH}}$ neurons in GAD65::Cre mice by injecting the vector AAV8.hSvn-DIO-hm3D(Gq)-mCherry $\left(2.2 \times 10^{12}\right.$ genome copies $(\mathrm{gc}) / \mathrm{mL}$; UNC Vector Core) into LH of the GAD65::Cre mice ${ }^{16}$.

Fiber photometry. After LH injection of MCH-promoter-driven GCaMP6s, alone in C57/Bl6 mice or in combination with the Cre-dependent activatory DREADD (hM3Dq) in GAD65::Cre mice, fiberoptic implants were stereotaxically installed with the fiber tip above the $\mathrm{LH}(1.35 \mathrm{~mm}$ caudal from bregma, $1.0 \mathrm{~mm}$ lateral from midline, and $5 \mathrm{~mm}$ ventral from brain surface) and fixed to the skull as in our previous work ${ }^{32,13}$. This method is estimated to capture fluorescence signals from within $\approx 500 \mu \mathrm{m}$ of the fiber tip ${ }^{32}$. Fiber tip locations were verified in each mouse by examining slices with a visible fiber tract. During fiber photometry experiments ${ }^{32}$, the excitation mode was set to provide interleaved $405 \mathrm{~nm}$ and $470 \mathrm{~nm}$ excitation light pulses via LEDs ${ }^{33}$. Fluorescence emission produced by $405 \mathrm{~nm}$ excitation is not sensitive to calcium and thus provides a real-time control for motion artefacts $^{33}$. Fluorescence signals were normalized to produce the plotted $\% \Delta F / F$ values as follows: $\Delta F / F=100^{*}(\mathrm{Fr}-F) / F$, where $\mathrm{Fr}$ is the raw signal and $F$ is the mean of the first $10 \mathrm{~s}$ of trial. Before photometry recordings, mice were habituated to the recording chamber, the plugging in procedure, and (where relevant) i.p. injections. On the day of fiber photometry recordings, mice were given $10 \mathrm{~min}$ to adjust to the chamber before an object was introduced. During the next $1 \mathrm{~h}$ mice had time to familiarize themselves with the object, after which the object was removed (i.p. injections of $\mathrm{CNO}$ or saline where performed where relevant at this point). Thirty minutes after this, the familiar object was reintroduced and mice had free access to explore it while their brain signals were recorded, and head location was videotracked (Ethovision XP, 15 frames/s). The novel object trial followed, by exchanging the familiar object for a novel object and allowing mice to freely explore the novel object while their brain signals were recorded, and head location was videotracked. Object exploration bouts were detected by nose video-tracking (Ethovision $\mathrm{XP}$ ), and their onset defined as the first frame when the mouse nose entered the object area (defined as a $3 \mathrm{~cm}$-wide perimeter around the object). Choice of objects for familiar and novel trials were based on a crossover design to avoid any confounding factors due to differences in objects. To compare MCH::GCaMP6 $\mathrm{s}_{\mathrm{LH}}$ calcium signals between mice, we selected the first 10 exploration bouts of each mouse for the novel and familiar objects, and used these data to derive averaged signal per mouse. During the first 10 entries the object was investigated more frequently if it was novel (Supplementary Fig. $2 \mathrm{H}$ ), and since our aim was to define neural correlates of behavioral responses to novel objects, we chose the first 10 entries for analysis of $\mathrm{MCH}$ photometry signals.

Closed-loop neural optosilencing. Mice were bilaterally LH-injected with Credependent ArchT (or, in control experiments, Cre-dependent GCaMP6s), and bilaterally implanted with intra-LH optical fibers using the coordinates and procedures as described above for fiber photometry. Three weeks after surgery, mice were handled and habituated to the recording arena before any procedure started. For experiments, a green laser ( $532 \mathrm{~nm}$, LaserGlow) was connected to the bilateral fibre implants to yield $\approx 20 \mathrm{~mW}$ light power output at the fiber tip. Since photometry recordings showed an onset of increased $\mathrm{MCH}$ neuron activity before mice entered the object area (Fig. 1d), we paired bilateral LH laser illumination with times when mouse nose was $<2 \mathrm{~cm}$ away from object area (i.e., within $5 \mathrm{~cm}$ perimeter from object). For control experiments investigating the effect of silencing $\mathrm{GAD}_{\mathrm{LH}}$ or $\mathrm{MCH}_{\mathrm{LH}}$ neurons on object exploration, mice were freely behaving for $10 \mathrm{~min}$ in an open field arena with two identical objects, and the peri-object area of one object (defined as above) was paired with the bilateral LH laser illumination to 
test for the $\mathrm{GAD}_{\mathrm{LH}}$ or $\mathrm{MCH}_{\mathrm{LH}}$ neuron effects on object exploration (Supplementary Fig. 2A-D). The propensities for self-paced object investigation of GAD65::Cre and MCH::Cre mice were investigated in control experiments and found to be similar (Supplementary Fig. 2I).

Object recognition memory tests. For object recognition memory tests ${ }^{15}$ (Figs. 2c, d; 4c-e), during the laser ON familiarisation, the bilateral LH laser illumination was triggered whenever the mouse entered the peri-object area (as defined above) of either object. No laser was applied during recognition trials. Laser OFF familiarisation was performed in the same mice with the same temporal contingencies as laser ON familiarisation, but with a new set of objects. After $1 \mathrm{~h}$ of retention interval, during which mice were returned to their home cages and no experimental manipulations were performed, the recognition trial $(=$ trial 2$)$ consisted of $10 \mathrm{~min}$ during which mice freely explored one object from the previous familiarisation trial (familiar object) and one novel object. Sets of novel and familiar objects were alternated between mice in a crossover design. For novel object recognition tests where $\mathrm{MCH}$ receptors were blocked with SNAP 94847 (Fig. $4 \mathrm{c}-\mathrm{e}$ ), mice were i.p. injected with SNAP or vehicle solutions $45 \mathrm{~min}$ before trial 1. In these experiments, a longer interval between familiarisation/acquisition and recognition trials was used $(20 \mathrm{~h})$, to ensure that $\mathrm{MCH}$ receptors were only blocked during memory acquisition, and that mice were unimpaired by SNAP during recognition tests.

Our aim was to specifically examine the effects of LH optosilencing on memory formation, independently of factors such as the duration of sensory exposure to objects during familiarisation/memorisation. Therefore, in laser ON and laser OFF familiarization trials, a constant cumulative exposure of mice to objects was imposed, by real-time video tracking of the cumulative object encounter time (time when the mouse nose was in the object area), and terminating all trials when the same cumulative object encounter time ( $30 \mathrm{~s})$ was reached. This ensured that differences in object memory acquisition were not due to variation in initial object exposure between different mice or trials, or different optosilencing conditions that may otherwise have influenced the total object investigation time as suggested by our control experiments (Supplementary Fig. 2A-D).

Y maze test of spatial memory. Continuous spontaneous alterations in a Y maze were measured with and without concurrent optogenetic silencing in the same mice (sequence of optogenetic silencing and laser off was alternated between mice) (Supplementary Fig. 4). Mice were connected to bilateral patch cords 10 min before start of the experiments and then transferred to the center of a standard $\mathrm{Y}$ maze (3 arms, $30 \mathrm{~cm}$ long, $120^{\circ}$ apart). During the following $8 \mathrm{~min}$, mice were free to explore the arms of the Y maze whilst video tracking with Noldus Ethovision scored the spontaneous alterations defined as consecutive entries into three different $\operatorname{arms}^{34,35}$

Experimental sequences in behavioral experiments. Crossover-like experimental designs were used in all in vivo photometry and optogenetic experiments, to prevent artefacts and biases and isolate the effects of variables under investigation. Specifically, presentations of novel and familiar object were alternated within and between mice to avoid behavioral fatigue or order effects. Photometry experiments were designed to expose the same mouse to sequences of novel and familiar objects that avoided behavioral habituation or calcium indicator degradation as confounding factors (e.g., novel $\rightarrow$ familiar $\rightarrow$ novel, Fig. 1d, Supplementary Fig. 1B, C). Optogenetic experiments were based on a crossover-like design where manipulations involving drugs, laser light, or mouse genotype were arranged in a Latin square to avoid any confounding factors due to day to day differences or carry-over effects. To prevent potential arena side biases from influencing the results of experiments involving two objects positioned at different sides of arena, trials were repeated with laser OFF and ON sides reversed; the presented results are an average of both trials.

Channelrhodopsin-assisted circuit mapping in brain slices. For brain slice patch-clamp recordings combined with optogenetics ${ }^{16,36}$, LH slices were prepared at least 2 months after virus injection. Coronal brain slices containing the LH were cut at $250 \mu \mathrm{m}$ thickness while immersed in ice-cold slicing solution. Slices were incubated for $1 \mathrm{~h}$ in artificial cerebrospinal fluid (ACSF) at $35^{\circ} \mathrm{C}$, and then transferred to a submerged-type recording chamber. Neurons containing fluorescent markers were visualized with an Olympus BX61WI microscope with an oblique condenser and fluorescence filters. Excitation light was delivered from a LAMBDA DG-5 beam switcher (Sutter) with a xenon lamp and ET470/40 (for ChR2) or ET500/20 (for ArchT) bandpass filters. A 40× 0.8NA objective was used to deliver pulses of excitation light $\left(\sim 10 \mathrm{~mW} / \mathrm{mm}^{2}, 1 \mathrm{~ms}\right.$ for ChR2 activation, or $1 \mathrm{~s}$ for ArchT activation) around the recorded cell, and postsynaptic responses were recorded in voltage-clamp (for circuit mapping) or current-clamp (for confirmation of ArchT-mediated photinhibition). Functional ChR2 expression was confirmed by recording light-activated action potentials in the target cells $(n=3$ cells per group, not shown). For testing LH output connections of GAD65 ${ }_{\mathrm{LH}}$ cells, we chose LH neurons based on their genetic markers (MCH::GFP, orexin::GFP,
GAD65::GFP) without noting GAD65 axon location. However, GAD65 axons were dense and abundant everywhere in the $\mathrm{LH}^{16}$.

Chemicals and solutions. For brain slice recordings, ACSF and ice-cold slicing solution were gassed with $95 \% \mathrm{O}_{2}$ and $5 \% \mathrm{CO}_{2}$, and contained the following: $125 \mathrm{mM} \mathrm{NaCl}$ ACSF, $2.5 \mathrm{mM} \mathrm{KCl}, 1 \mathrm{mM} \mathrm{MgCl} 2,2 \mathrm{mM} \mathrm{CaCl}, 1.2 \mathrm{mM} \mathrm{NaH}_{2} \mathrm{PO}_{4}$, $21 \mathrm{mM} \mathrm{NaHCO}, 2 \mathrm{mM} \mathrm{D}$-(+)-glucose, $0.1 \mathrm{mM} \mathrm{Na}+$-pyruvate, and $0.4 \mathrm{mM}$ ascorbic acid. The slicing solution contained $2.5 \mathrm{mM} \mathrm{KCl}, 1.3 \mathrm{mM} \mathrm{NaH}{ }_{2} \mathrm{PO} \cdot \mathrm{H}_{2} \mathrm{O}$, $26.0 \mathrm{mM} \mathrm{NaHCO} 3,213.3 \mathrm{mM}$ sucrose, $10.0 \mathrm{mM} \mathrm{D}$-(+)-glucose, $2.0 \mathrm{mM} \mathrm{MgCl}$ and $2.0 \mathrm{mM} \mathrm{CaCl}_{2}$. For standard whole-cell recordings, pipettes were filled with intracellular solution containing the following: $120 \mathrm{mM}$ K-gluconate, $10 \mathrm{mM} \mathrm{KCl}$, $10 \mathrm{mM}$ Hepes, $0.1 \mathrm{mM}$ EGTA, $4 \mathrm{mM}$ K2ATP, $2 \mathrm{mM} \mathrm{Na} 2 \mathrm{ATP}, 0.3 \mathrm{mM} \mathrm{Na} 2 \mathrm{GTP}$ and $2 \mathrm{mM} \mathrm{MgCl}_{2}(\mathrm{pH} 7.3)$ with KOH. Gabazine $(3 \mu \mathrm{m})$ was used where indicated. For in vivo chemogenetic manipulations, $\mathrm{CNO}$ was injected i.p. at $0.5 \mathrm{mg} / \mathrm{kg}$ body weight in experiments involving hM3Dq. The MCH receptor antagonist SNAP 94847 hydrochloride was injected i.p. at $20 \mathrm{mg} / \mathrm{kg}$ body weight (based on ref. ${ }^{37}$ ) after being dissolved in distilled water with $10 \%$ DMSO and $30 \mathrm{mg} / \mathrm{ml}$ (2-Hydroxypropyl)- $\beta$-cyclodextrin. All chemicals were from Sigma or Tocris Bioscience.

Immunohistochemistry. For the immunolabeling of $\mathrm{MCH}$ neurons, $50-\mu \mathrm{m}$ cryosections of pMCH-dependent GCaMP6s injected C57B/16 mice were stained for MCH with a rabbit antibody to MCH (H-070-47,1:2000, Phoenix Pharmaceuticals) as a primary antibody, and Alexa 555-conjugated donkey antibody to rabbit IgG (A-21244, 1:500, Invitrogen) as a secondary antibody. Slices were then imaged with an Olympus VS120 slide scanner microscope and double labelling of GCaMP with Alexa 555 was quantified with ImageJ.

Statistical analyses. Statistical tests and descriptive statistics were performed as specified in the figure legends. All experimental animals were included in the analyses (no pre-selection or exclusion). In each experimental dataset at the cellular level, each $\mathrm{n}$ was a different cell (no repeated trials from the same cell were used as $n$ values) and cells from at least three mice were analyzed. Before performing parametric tests, data were assessed for normality with a D'Agostino-Pearson omnibus test or Kolmogorov-Smirnov test for small sample sizes. To compare interactions within normally distributed data with repeated measurements, repeated measures ANOVA was used, with multiple comparison tests where appropriate. All statistical tests are two tailed unless otherwise stated. All error bars indicate the standard error of the mean. Analysis was performed with GraphPad Prism and MATLAB (The MathWorks, Inc.).

Reporting summary. Further information on research design is available in the Nature Research Reporting Summary linked to this article.

\section{Data availability}

The datasets generated and analyzed during the current study are available from the corresponding author on reasonable request.

\section{Code availabilty}

Custom codes for the acquisition of photometry data (Labview) and for the analysis of photometry data (Matlab) are available from the corresponding author on reasonable request.

Received: 8 April 2019 Accepted: 8 May 2019

Published online: 07 June 2019

\section{References}

1. Aggleton, J. P. \& Shaw, C. Amnesia and recognition memory: a re-analysis of psychometric data. Neuropsychologia 34, 51-62 (1996).

2. Winters, B. D., Saksida, L. M. \& Bussey, T. J. Object recognition memory: neurobiological mechanisms of encoding, consolidation and retrieval. Neurosci. Biobehav. Rev. 32, 1055-1070 (2008).

3. Broadbent, N. J., Gaskin, S., Squire, L. R. \& Clark, R. E. Object recognition memory and the rodent hippocampus. Learn Mem. 17, 5-11 (2010).

4. Squire, L. R., Wixted, J. T. \& Clark, R. E. Recognition memory and the medial temporal lobe: a new perspective. Nat. Rev. Neurosci. 8, 872-883 (2007).

5. Ishii, T. Distribution of Alzheimer's neurofibrillary changes in the brain stem and hypothalamus of senile dementia. Acta Neuropathol. 6, 181-187 (1966).

6. Saper, C. B. \& German, D. C. Hypothalamic pathology in Alzheimer's disease. Neurosci. Lett. 74, 364-370 (1987).

7. Schwartz, M. \& Teitelbaum, P. Dissociation between learning and remembering in rats with lesions in the lateral hypothalamus. J. Comp. Physiol. Psychol. 87, 384-398 (1974). 
8. Adamantidis, A. \& de Lecea, L. A role for Melanin-Concentrating Hormone in learning and memory. Peptides 30, 2066-2070 (2009).

9. Pachoud, B. et al. Major impairments of glutamatergic transmission and longterm synaptic plasticity in the hippocampus of mice lacking the melaninconcentrating hormone receptor-1. J. Neurophysiol. 104, 1417-1425 (2010).

10. Bittencourt, J. C. et al. The melanin-concentrating hormone system of the rat brain: an immuno- and hybridization histochemical characterization. J. Comp. Neurol. 319, 218-245 (1992).

11. Bittencourt, J. C. Anatomical organization of the melanin-concentrating hormone peptide family in the mammalian brain. Gen. Comp. Endocrinol. 172, 185-197 (2011).

12. Hassani, O. K., Lee, M. G. \& Jones, B. E. Melanin-concentrating hormone neurons discharge in a reciprocal manner to orexin neurons across the sleepwake cycle. Proc. Natl Acad. Sci. USA 106, 2418-2422 (2009).

13. Gonzalez, J. A., Iordanidou, P., Strom, M., Adamantidis, A. \& Burdakov, D. Awake dynamics and brain-wide direct inputs of hypothalamic $\mathrm{MCH}$ and orexin networks. Nat. Commun. 7, 11395 (2016).

14. Ennaceur, A. \& Delacour, J. A new one-trial test for neurobiological studies of memory in rats. 1: Behavioral data. Behav. Brain Res 31, 47-59 (1988).

15. Bevins, R. A. \& Besheer, J. Object recognition in rats and mice: a one-trial non-matching-to-sample learning task to study 'recognition memory'. Nat. Protoc. 1, 1306-1311 (2006).

16. Kosse, C., Schone, C., Bracey, E. \& Burdakov, D. Orexin-driven GAD65 network of the lateral hypothalamus sets physical activity in mice. Proc. Natl Acad. Sci. USA 114, 4525-4530 (2017).

17. Antunes, M. \& Biala, G. The novel object recognition memory: neurobiology, test procedure, and its modifications. Cogn. Process 13, 93-110 (2012).

18. Adamantidis, A. et al. Disrupting the melanin-concentrating hormone receptor 1 in mice leads to cognitive deficits and alterations of NMDA receptor function. Eur. J. Neurosci. 21, 2837-2844 (2005).

19. Monzon, $\mathrm{M}$. E. et al. Melanin-concentrating hormone $(\mathrm{MCH})$ modifies memory retention in rats. Peptides 20, 1517-1519 (1999).

20. Dietrich, M. O., Zimmer, M. R., Bober, J. \& Horvath, T. L. Hypothalamic Agrp neurons drive stereotypic behaviors beyond feeding. Cell 160, 1222-1232 (2015).

21. Sharpe, M. J. et al. Lateral hypothalamic GABAergic neurons encode reward predictions that are relayed to the ventral tegmental area to regulate learning. Curr. Biol. 27, 2089-2100 e2085 (2017).

22. Zimmer, M. R., Schmitz, A. E. \& Dietrich, M. O. Activation of Agrp neurons modulates memory-related cognitive processes in mice. Pharm. Res. 141, 303-309 (2019).

23. Burnett, C. J. et al. Hunger-Driven Motivational State Competition. Neuron 92, 187-201 (2016).

24. Jego, S. et al. Optogenetic identification of a rapid eye movement sleep modulatory circuit in the hypothalamus. Nat. Neurosci. 16, 1637-1643 (2013).

25. Hausen, A. C. et al. Insulin-dependent activation of $\mathrm{MCH}$ neurons impairs locomotor activity and insulin sensitivity in obesity. Cell Rep. 17, 2512-2521 (2016).

26. Burdakov, D., Gerasimenko, O. \& Verkhratsky, A. Physiological changes in glucose differentially modulate the excitability of hypothalamic melaninconcentrating hormone and orexin neurons in situ. J. Neurosci. 25, 2429-2433 (2005).

27. Kong, D. et al. Glucose stimulation of hypothalamic $\mathrm{MCH}$ neurons involves $\mathrm{K}$ (ATP) channels, is modulated by UCP2, and regulates peripheral glucose homeostasis. Cell Metab. 12, 545-552 (2010).

28. Taniguchi, $\mathrm{H}$. et al. A resource of Cre driver lines for genetic targeting of GABAergic neurons in cerebral cortex. Neuron 71, 995-1013 (2011).

29. Karnani, M. M., Szabo, G., Erdelyi, F. \& Burdakov, D. Lateral hypothalamic GAD65 neurons are spontaneously firing and distinct from orexin- and melanin-concentrating hormone neurons. J. Physiol. 591, 933-953 (2013).

30. Burdakov, D. et al. Tandem-pore $\mathrm{K}+$ channels mediate inhibition of orexin neurons by glucose. Neuron 50, 711-722 (2006).

31. Apergis-Schoute, J. et al. Optogenetic evidence for inhibitory signaling from orexin to MCH neurons via local microcircuits. J. Neurosci. 35, 5435-5441 (2015).
32. Gonzalez, J. A. et al. Inhibitory Interplay between Orexin Neurons and Eating. Curr. Biol. 26, 2486-2491 (2016).

33. Kim, C. K. et al. Simultaneous fast measurement of circuit dynamics at multiple sites across the mammalian brain. Nat. Methods 13, 325-328 (2016).

34. Wolf, A., Bauer, B., Abner, E. L., Ashkenazy-Frolinger, T. \& Hartz, A. M. A Comprehensive Behavioral Test Battery to Assess Learning and Memory in 129S6/Tg2576 Mice. PloS ONE 11, e0147733 (2016).

35. Miedel C. J., Patton J. M., Miedel A. N., Miedel E. S. \& Levenson J. M. Assessment of spontaneous alternation, novel object recognition and limb clasping in transgenic mouse models of amyloid-beta and tau neuropathology. J. Vis. Exp. (2017)

36. Schöne, C. et al. Optogenetic probing of fast glutamatergic transmission from hypocretin/orexin to histamine neurons in situ. J. Neurosci. 32, 12437-12443 (2012).

37. Marsteller, D. A. et al. The MCH1 receptor antagonist SNAP 94847 induces sensitivity to dopamine D2/D3 receptor agonists in rats and mice. Eur. J. Pharm. 602, 66-72 (2009).

\section{Acknowledgements}

The experiments in this study were supported by the Francis Crick Institute which receives its core funding from Cancer Research UK (FC001055), the UK Medical Research Council (FC001055), and the Wellcome Trust (FC001055). We thank Prof. Antoine Adamantidis, Dr. Daria Peleg-Raibstein, and Cristina Concetti for critical reading of parts of the manuscript; and Dr. Lauren Mulholland for advice on the writing. The authors have no competing interests. All data are available in the manuscript or the supplementary materials.

\section{Author contributions}

C.K. designed and carried out the experiments, analyzed data, and co-wrote the text. D.B. conceived the study, advised on experiments, and wrote the text.

\section{Additional information}

Supplementary Information accompanies this paper at https://doi.org/10.1038/s41467019-10484-7.

Competing interests: The authors declare no competing interests.

Reprints and permission information is available online at http://npg.nature.com/ reprintsandpermissions/

Journal peer review information: Nature Communications thanks Michael Krashes and the other, anonymous, reviewer(s) for their contribution to the peer review of this work.

Publisher's note: Springer Nature remains neutral with regard to jurisdictional claims in published maps and institutional affiliations.

\begin{abstract}
(c) (i) Open Access This article is licensed under a Creative Commons Attribution 4.0 International License, which permits use, sharing adaptation, distribution and reproduction in any medium or format, as long as you give appropriate credit to the original author(s) and the source, provide a link to the Creative Commons license, and indicate if changes were made. The images or other third party material in this article are included in the article's Creative Commons license, unless indicated otherwise in a credit line to the material. If material is not included in the article's Creative Commons license and your intended use is not permitted by statutory regulation or exceeds the permitted use, you will need to obtain permission directly from the copyright holder. To view a copy of this license, visit http://creativecommons.org/ licenses/by/4.0/.
\end{abstract}

(C) The Author(s) 2019 